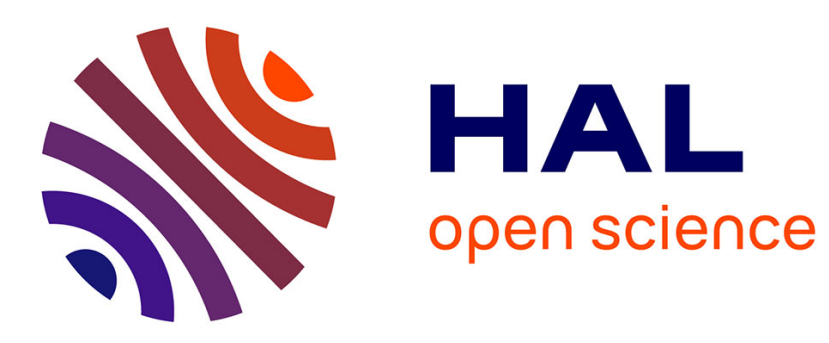

\title{
On a Bloch-type model with electron-phonon interactions: modeling and numerical simulations
}

Brigitte Bidégaray-Fesquet, Clément Jourdana, Kole Keita

\section{To cite this version:}

Brigitte Bidégaray-Fesquet, Clément Jourdana, Kole Keita. On a Bloch-type model with electronphonon interactions: modeling and numerical simulations. 12th International Conference on Scientific Computing in Electrical Engineering (SCEE 2018), Sep 2018, Taormina, Italy. pp.105-113, 10.1007/978-3-030-44101-2_10. hal-02043321

\section{HAL Id: hal-02043321 \\ https://hal.univ-grenoble-alpes.fr/hal-02043321}

Submitted on 20 Feb 2019

HAL is a multi-disciplinary open access archive for the deposit and dissemination of scientific research documents, whether they are published or not. The documents may come from teaching and research institutions in France or abroad, or from public or private research centers.
L'archive ouverte pluridisciplinaire HAL, est destinée au dépôt et à la diffusion de documents scientifiques de niveau recherche, publiés ou non, émanant des établissements d'enseignement et de recherche français ou étrangers, des laboratoires publics ou privés. 


\title{
On a Bloch-type model with electron-phonon interactions: modeling and numerical simulations
}

\author{
B. Bidégaray-Fesquet, C. Jourdana, and K. Keita
}

\begin{abstract}
In this work, we discuss how to take into account electron-phonon interactions in a Bloch type model for the description of quantum dots. The model consists in coupling an equation on the density matrix with a set of equations on quantities called phonon-assisted densities, one for each phonon mode. After a description of the model, we discuss how to discretize efficiently this non-linear coupling in view of numerical simulations.
\end{abstract}

\section{Bloch model}

\subsection{Quantum dot description}

Quantum dots are usually described using electrons and holes. As detailed in [2], we prefer a conduction and valence electron description, where valence electrons can be seen as an absence of holes in a valence band. Due to the 3D confinement, energy levels are quantized for each species of electrons and can be indexed by integers. We denote respectively $\left(\varepsilon_{j}^{c}\right)_{j \in \mathscr{I}^{c}}$ and $\left(\varepsilon_{j}^{v}\right)_{j \in \mathscr{I}^{v}}$ the conduction and valence energy levels.

To describe the time evolution of the energy level occupations, we define a global density matrix by

$$
\rho=\left(\begin{array}{cc}
\rho^{c} & \rho^{c v} \\
\rho^{v c} & \rho^{v}
\end{array}\right)
$$

B. Bidégaray-Fesquet, C. Jourdana

Univ. Grenoble Alpes, CNRS, Grenoble INP ${ }^{\dagger}$, LJK, 38000 Grenoble, France

$\dagger$ Institute of Engineering Univ. Grenoble Alpes

e-mail: brigitte.bidegaray@univ-grenoble-alpes.fr, clement.jourdana@univ-grenoble-alpes.fr

K. Keita

Univ. Jean Lorougnon Guédé (UJLoG), 2 BP V 25 DALOA 12, Ivory Coast

e-mail: keitakole96@gmail.com 
The matrices $\rho^{c}$ and $\rho^{v}$ are respectively the conduction and valence densities. Their diagonal terms, called populations, are the occupation probabilities and their offdiagonal terms, called coherences, describe the intra-band transitions. Finally, $\rho^{c v}$ and $\rho^{v c}=\rho^{c v^{*}}\left(A^{*}\right.$ denoting the Hermitian adjoint of a matrix $\left.A\right)$ describe the interband transitions.

The time-evolution of $\rho$ can be driven by a free electron Hamiltonian associated to electron level energies and the interaction with an electromagnetic wave (see e.g. [2] for details):

$$
i \hbar \partial_{t} \rho=\left[E_{0}+\mathbf{E} \cdot \mathbf{M}, \rho\right],
$$

where $[A, B]$ denotes the commutator $A B-B A, E_{0}=\operatorname{diag}\left(\left\{\varepsilon_{j}^{c}\right\},\left\{\varepsilon_{j}^{v}\right\}\right), \mathbf{M}$ is the dipolar moment matrix (a matrix that can be expressed in terms of the wave functions associated to each energy level) and $\mathbf{E}$ is a time-dependent electric field.

To study the interaction of the quantum dot with an electromagnetic field, equation (2) can be coupled with Maxwell equations:

$$
\begin{aligned}
& \partial_{t} \mathbf{E}=c^{2} \operatorname{curl} \mathbf{B}-\mu_{0} c^{2} \mathbf{J}, \\
& \partial_{t} \mathbf{B}=-\operatorname{curl} \mathbf{E},
\end{aligned}
$$

B being the magnetic field, $c$ the speed of light in free space and $\mu_{0}$ the vacuum permeability. The coupling is expressed via the current density $\mathbf{J}$ which is given by

$$
\mathbf{J}=n_{a} \operatorname{Tr}\left(\mathbf{M} \partial_{t} \rho\right),
$$

where $n_{a}$ is the quantum dot volume density.

Equation (2) is a Liouville equation and it confers a certain number of properties to the solution that have already been extensively studied in the literature. Here, we focus on the addition of electron-phonon (e-ph) interactions in such a model.

\subsection{Electron-phonon Hamiltonian}

As in [3] where the addition of Coulomb interactions is discussed, the starting point is to use field quantification to write an e-ph Hamiltonian. We write it in the form $H^{c-p h}+H^{v-p h}$. It reflects that e-ph interactions cannot lead the electron to change species. In this work, only polar coupling to optical phonons is considered since it usually leads to the fastest dynamics in low excitation regime. The corresponding Fröhlich interaction Hamiltonian is given by (see e.g. $[4,6]$ ):

$$
\begin{aligned}
H^{c-p h} & =\frac{1}{|\mathscr{B}|} \int_{\mathscr{B}} \sum_{\alpha, \alpha^{\prime} \in \mathscr{I}^{c}} G_{\mathbf{q}, \alpha, \alpha^{\prime}}^{c} c_{\alpha}^{\dagger}\left(b_{\mathbf{q}}+b_{-\mathbf{q}}^{\dagger}\right) c_{\alpha^{\prime}} \mathrm{d} \mathbf{q}, \\
H^{v-p h} & =\frac{1}{|\mathscr{B}|} \int_{\mathscr{B}} \sum_{\alpha, \alpha^{\prime} \in \mathscr{I}^{v}} G_{\mathbf{q}, \alpha, \alpha^{\prime}}^{v} v_{\alpha}^{\dagger}\left(b_{\mathbf{q}}+b_{-\mathbf{q}}^{\dagger}\right) v_{\alpha^{\prime}} \mathrm{d} \mathbf{q} .
\end{aligned}
$$


The operators $c_{j}^{\dagger}$ and $c_{j}$ (resp. $v_{j}^{\dagger}$ and $v_{j}$ ) are creation and annihilation operators for conduction (resp. valence) electrons and the operators $b_{\mathbf{q}}^{\dagger}$ and $b_{\mathbf{q}}$ are those for phonons, where the phonon mode $\mathbf{q}$ belongs to the Brillouin zone $\mathscr{B}$ of the underlying crystal. The volume of the Brillouin zone is denoted $|\mathscr{B}|$. For $e \in\{c, v\}, G_{\mathbf{q}}^{e}$ is a matrix whose coefficients are expressed in terms of the wave functions associated to each energy level:

$$
G_{\mathbf{q}, \alpha, \alpha^{\prime}}^{e}=\mathscr{E}_{\mathbf{q}} \int \psi_{\alpha}^{e *}(\mathbf{r}) \exp (i \mathbf{q} \cdot \mathbf{r}) \psi_{\alpha^{\prime}}^{e}(\mathbf{r}) \mathrm{d} \mathbf{r},
$$

$\mathscr{E}_{\mathbf{q}}$ being the Fröhlich constant [6] defined such that $G_{\mathbf{q}}^{e *}=G_{-\mathbf{q}}^{e}$.

\subsection{Phonon-assisted densities and time evolution}

We first recall that the commutation relations between conduction and valence electron operators are the following

$$
\begin{array}{rrr}
\forall j, k \in \mathscr{I}^{c}, & \left\{c_{j}^{\dagger}, c_{k}\right\}=\delta_{j k}, & \left\{c_{j}^{\dagger}, c_{k}^{\dagger}\right\}=\left\{c_{j}, c_{k}\right\}=0, \\
\forall j, k \in \mathscr{I}^{v}, & \left\{v_{j}, v_{k}^{\dagger}\right\}=\delta_{j k}, & \left\{v_{j}, v_{k}\right\}=\left\{v_{j}^{\dagger}, v_{k}^{\dagger}\right\}=0, \\
\forall j \in \mathscr{I}^{c} \text { and } k \in \mathscr{I}^{v}, & {\left[c_{j}^{\dagger}, v_{k}^{\dagger}\right]=\left[c_{j}^{\dagger}, v_{k}\right]=\left[c_{j}, v_{k}^{\dagger}\right]=\left[c_{j}, v_{k}\right]=0,}
\end{array}
$$

where $\delta_{j k}$ is the Kronecker delta function. Contrarily to electrons, phonons are bosons and they obey the following commutation rules

$$
\forall \mathbf{q}, \mathbf{q}^{\prime} \in \mathscr{B}, \quad\left[b_{\mathbf{q}}, b_{\mathbf{q}^{\prime}}^{\dagger}\right]=|\mathscr{B}| \boldsymbol{\delta}\left(\mathbf{q}-\mathbf{q}^{\prime}\right), \quad\left[b_{\mathbf{q}}, b_{\mathbf{q}^{\prime}}\right]=\left[b_{\mathbf{q}}^{\dagger}, b_{\mathbf{q}^{\prime}}^{\dagger}\right]=0,
$$

where $\delta$ is the Dirac delta function. Indeed, we do not consider here a quantization of phonon modes (even if in order to perform numerical simulations we will discretize in Section 2 the Brillouin zone and use a quadrature formula to approximate integrals over $\mathbf{q})$.

As in [5], we introduce phonon-assisted density matrices

$$
S_{\mathbf{q}}=\left(\begin{array}{ll}
S_{\mathbf{q}}^{c c} & S_{\mathbf{q}}^{c v} \\
S_{\mathbf{q}}^{c c} & S_{\mathbf{q}}^{v v}
\end{array}\right)
$$

where $S_{\mathbf{q}, \alpha, \alpha^{\prime}}^{e f}=\left\langle f_{\alpha^{\prime}}^{\dagger} b_{\mathbf{q}} e_{\alpha}\right\rangle, e, f \in\{c, v\}$. Then, using intensively the commutativity rules (9)-(12), the time evolution of the density matrix due to e-ph interactions can be cast in a very compact form as

$$
\left.i \hbar \partial_{t} \rho\right|_{e-p h}=\frac{1}{|\mathscr{B}|} \int_{\mathscr{B}}\left[G_{\mathbf{q}}, S_{\mathbf{q}}+S_{-\mathbf{q}}^{*}\right] \mathrm{d} \mathbf{q} \equiv P(S),
$$

where we have introduced the notations 


$$
G_{\mathbf{q}}=\left(\begin{array}{cc}
G_{\mathbf{q}}^{c} & 0 \\
0 & G_{\mathbf{q}}^{v}
\end{array}\right) \text { and } S=\left\{S_{\mathbf{q}}, \mathbf{q} \in \mathscr{B}\right\}
$$

We have $P(S)^{*}=-P(S)$ independently of the structure of $S$. Therefore $\rho$, which is initially Hermitian, remains Hermitian through time evolution via (14). This equation is also trace preserving since the right-hand side is a combination of trace-free commutators.

To close the system, we now look for the time evolution of phonon-assisted densities. First, using again the commutativity rules (9)-(12), we make explicit the commutators between the e-ph interaction Hamiltonian and the other Hamiltonians involved in the system: the free electron Hamiltonian, the electromagnetic interaction Hamiltonian and the free phonon Hamiltonian. For instance, the free phonon Hamiltonian is given by

$$
H^{\mathrm{ph}}=\frac{1}{|\mathscr{B}|} \int_{\mathscr{B}} E_{\mathbf{q}} b_{\mathbf{q}}^{\dagger} b_{\mathbf{q}} \mathrm{d} \mathbf{q}
$$

and the involved commutator $\left[c_{\alpha}^{\dagger}\left(b_{\mathbf{q}}+b_{-\mathbf{q}}^{\dagger}\right) c_{\alpha^{\prime}}, H^{\mathrm{ph}}\right]$ is equal to $E_{\mathbf{q}} c_{\alpha}^{\dagger}\left(b_{\mathbf{q}}-b_{-\mathbf{q}}^{\dagger}\right) c_{\alpha^{\prime}}$. Then, we use the Wick theorem [8] to approximate the means involving four operators by sums and products of densities. It closes the system at the cost of rendering it non-linear. After computations, we finally obtain, for each $\mathbf{q} \in \mathscr{B}$, the following equation

$$
\begin{aligned}
\left.i \hbar \partial_{t} S_{\mathbf{q}}\right|_{e-p h} & =E_{\mathbf{q}} S_{\mathbf{q}}+\frac{1}{2}\left\{G_{\mathbf{q}}^{*}, \rho\right\}+\left(\frac{1}{2}+n_{\mathbf{q}}\right)\left[G_{\mathbf{q}}^{*}, \rho\right]+C\left(\rho, G_{\mathbf{q}}^{*}\right) \\
& \equiv E_{\mathbf{q}} S_{\mathbf{q}}+Q_{\mathbf{q}}(\rho) .
\end{aligned}
$$

The term $\{A, B\}$ denotes the skew-commutator $A B+B A, n_{\mathbf{q}}=\left\langle b_{\mathbf{q}}^{\dagger} b_{\mathbf{q}}\right\rangle$ is the phonon density expressed in terms of the phonon energy $E_{\mathbf{q}}$ by the Bose-Einstein statistics, and $C\left(\rho, G_{\mathbf{q}}^{*}\right)$ is a non-linear term expressed as

$$
C\left(\rho, G_{\mathbf{q}}^{*}\right)=-\widetilde{\rho} G_{\mathbf{q}}^{*} \widetilde{\rho}+\operatorname{Tr}\left(G_{\mathbf{q}}^{*} \widetilde{\rho}\right) \widetilde{\rho}
$$

where $\widetilde{\rho}=\rho\left(\begin{array}{cc}I^{c} & 0 \\ 0 & -I^{v}\end{array}\right), I^{c}$ and $I^{v}$ being the identity matrices for the conduction and valence spaces.

To summarize, the e-ph Bloch model consists in coupling an equation on $\rho$

$$
i \hbar \partial_{t} \rho=\left[E_{0}+\mathbf{E} \cdot \mathbf{M}, \rho\right]+P(S)
$$

with a set of equations on $S_{\mathbf{q}}$ (one for each $\mathbf{q}$ )

$$
i \hbar \partial_{t} S_{\mathbf{q}}=E_{\mathbf{q}} S_{\mathbf{q}}+\left[E_{0}+\mathbf{E} \cdot \mathbf{M}, S_{\mathbf{q}}\right]+Q_{\mathbf{q}}(\rho) .
$$




\section{Numerical scheme}

For simulations, we consider a collection of quantum dots which are scattered in a one dimensional space along the $z$ direction and interact not directly but through the interaction with the electromagnetic field. Therefore, densities depend on time and space and the e-ph Bloch model (18)-(19) is coupled with Maxwell equations (3)-(4).

First, we introduce a uniform discretization of the Brillouin zone $\mathscr{B}$ using $N_{q}$ points. The integral over $\mathbf{q}$ in (14) is approximated by a simple quadrature formula and consequently the global phonon-assisted density $S=\left\{S_{\mathbf{q}_{l}}, l=1, \cdots, N_{q}\right\}$ is computed solving $N_{q}$ independent equations (19).

We fix a time step $\delta t>0$ and we discretize uniformly the time using $N_{t}+1$ points: $t_{n}=n \delta t$ for $n \in\left\{0, \ldots, N_{t}\right\}$ with $\delta t=\frac{T}{N_{t}}, T$ being the final time. Analogously, we fix a space step $\delta z>0$ and we discretize uniformly the space using $N_{z}+1$ points: $z_{j}=j \delta z$ for $j \in\left\{0, \ldots, N_{z}\right\}$ with $\delta z=\frac{L}{N_{z}}, L$ being the length of the quantum dot collection.

A finite difference Yee scheme is used for Maxwell equations (3)-(4): for all $n \in\left\{0, \cdots, N_{t}-1\right\}$,

$$
\begin{aligned}
B_{y, j+\frac{1}{2}}^{n+\frac{1}{2}} & =B_{y, j+\frac{1}{2}}^{n-\frac{1}{2}}-\frac{\delta t}{\delta z}\left(E_{x, j+1}^{n}-E_{x, j}^{n}\right), \quad \forall j \in\left\{0, \cdots, N_{z}-1\right\}, \\
E_{x, j}^{n+1} & =E_{x, j}^{n}-c^{2} \frac{\delta t}{\delta z}\left(B_{y, j+\frac{1}{2}}^{n+\frac{1}{2}}-B_{y, j-\frac{1}{2}}^{n+\frac{1}{2}}\right)-\mu_{0} c^{2} \delta t J_{x, j}^{n+\frac{1}{2}}, \quad \forall j \in\left\{1, \cdots, N_{z}\right\}
\end{aligned}
$$

with $J_{x, j}^{n+\frac{1}{2}}=-\frac{i n_{a}}{\hbar} \operatorname{Tr}\left(M_{x}\left[E_{0}, \rho_{j}^{n+\frac{1}{2}}\right]+M_{x} P\left(S_{j}^{n}\right)\right)$. In (20), we use the convention $B_{y, j+\frac{1}{2}}^{-\frac{1}{2}}=0$ for all $j \in\left\{0, \cdots, N_{z}-1\right\}$ and (21) is initialized by $E_{x, j}^{0}$ and $S_{j}^{0}=0$ for all $j \in\left\{0, \cdots, N_{z}\right\}$. Finally, a time dependent incident wave $E_{i n c}$ is injected in the left part of the device by the boundary condition

$$
E_{x, 0}^{n+1}=E_{x, 1}^{n}+E_{i n c}^{n+1}-E_{i n c}^{n-\frac{\delta z}{c}}+\frac{1-c \frac{\delta t}{\delta z}}{1+c \frac{\delta t}{\delta z}}\left(E_{x, 0}^{n}-E_{x, 1}^{n+1}+E_{i n c}^{n+1-\frac{\delta z}{c}}-E_{\text {inc }}^{n}\right),
$$

for all $n \in\left\{0, \cdots, N_{t}-1\right\}$. Notice that the discretization steps $\delta t$ and $\delta z$ are chosen in order to satisfy the stability condition imposed by this numerical scheme (see e.g. [1]).

We consider a weak coupling between the Maxwell and Bloch equations. It means that $E$ and $\rho$ are not discretized at the same time to avoid a fixed point procedure. Equations (18)-(19) are discretized on a staggered grid in time and each equation is solved using a Strang splitting method: 
for all $l \in\left\{1, \cdots, N_{\mathbf{q}}\right\}$, for all $n \in\left\{0, \cdots, N_{t}-1\right\}$, for all $j \in\left\{0, \cdots, N_{z}\right\}$,

$$
\begin{aligned}
S_{\mathbf{q}_{l}, j}^{n+1}= & \mathscr{A}_{3}\left(\frac{\delta t}{2}, E_{\mathbf{q}_{l}} I\right) \mathscr{A}_{2}\left(\frac{\delta t}{2}, E_{0}+\frac{E_{x, j}^{n}+E_{x, j}^{n+1}}{2} M_{x}\right) \\
& \mathscr{A}_{1}\left(\delta t, Q_{\mathbf{q}_{l}}\left(\rho_{j}^{n+\frac{1}{2}}\right)\right) \mathscr{A}_{2}\left(\frac{\delta t}{2}, E_{0}+\frac{E_{x, j}^{n}+E_{x, j}^{n+1}}{2} M_{x}\right) \mathscr{A}_{3}\left(\frac{\delta t}{2}, E_{\mathbf{q}_{l}} I\right) S_{\mathbf{q}_{l}, j}^{n}, \\
\rho_{j}^{n+\frac{3}{2}}= & \mathscr{A}_{2}\left(\frac{\delta t}{2}, E_{0}+E_{x, j}^{n+1} M_{x}\right) \mathscr{A}_{1}\left(\delta t, P\left(S_{j}^{n+1}\right)\right) \mathscr{A}_{2}\left(\frac{\delta t}{2}, E_{0}+E_{x, j}^{n+1} M_{x}\right) \rho_{j}^{n+\frac{1}{2}} .
\end{aligned}
$$

Equations (22)-(23) are initialized by $S_{\mathbf{q}_{l}, j}^{0}=0$ and $\rho_{j}^{\frac{1}{2}}=\mathscr{A}_{2}\left(\frac{\delta t}{2}, E_{0}+E_{x, j}^{0} M_{x}\right) \rho_{j}^{0}$. In these expressions, $\mathscr{A}_{1}, \mathscr{A}_{2}$ and $\mathscr{A}_{3}$ are three semigroups defined by

$$
\mathscr{A}_{1}(t, B) A=A-\frac{i t}{\hbar} B, \quad \mathscr{A}_{2}(t, B) A=e^{-\frac{i t B}{\hbar}} A e^{\frac{i t B}{\hbar}} \quad \text { and } \quad \mathscr{A}_{3}(t, B) A=e^{-\frac{i t B}{\hbar}} A .
$$

They can be computed exactly using matrix exponential formulas. The advantage of this splitting is that it numerically preserves positiveness for each equation.

\section{Numerical simulations}

We now perform numerical simulations in order to assess the capability of the Bloch model to describe the interaction of quantum dots with an electromagnetic field. More precisely, we study a Self-Induced Transparency (SIT) case. It is a phenomenon that allows to obtain an exact population inversion with an unchanged electric field, using a light pulse resonant with the electron energy levels.

The propagating field $E_{\text {inc }}$ that we inject is a pulse with a specific envelope and a center frequency $\omega_{0}$. First, we recall some results obtained without e-ph interactions for two 3-level test cases with a conduction level and two valence levels (see [3] for more details). The energy between the conduction level and the first valence level corresponds to the field frequency $\omega_{0}$. In the first case (dashed line on the schematic representation Fig.1), the transition between the two valence levels is $2 \hbar \omega_{0}$. It is instead $\hbar \omega_{0}$ in the second case (solid line).

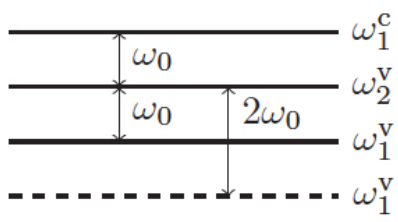

Fig. 1 Energy level description for the two 3-level test cases. 
In Fig.2, we represent at the top the time evolution of the electric field $E_{x}$ for a given quantum dot and at the bottom the corresponding time evolution of populations for the two test cases. In the left picture (corresponding to the case $\varepsilon_{2}^{v}-\varepsilon_{1}^{v}=2 \hbar \omega_{0}$ ), we observe a complete population inversion due to the light pulse. Instead, when the transition between the two valence levels is resonant with the field (right picture), the SIT phenomenon is destroyed.
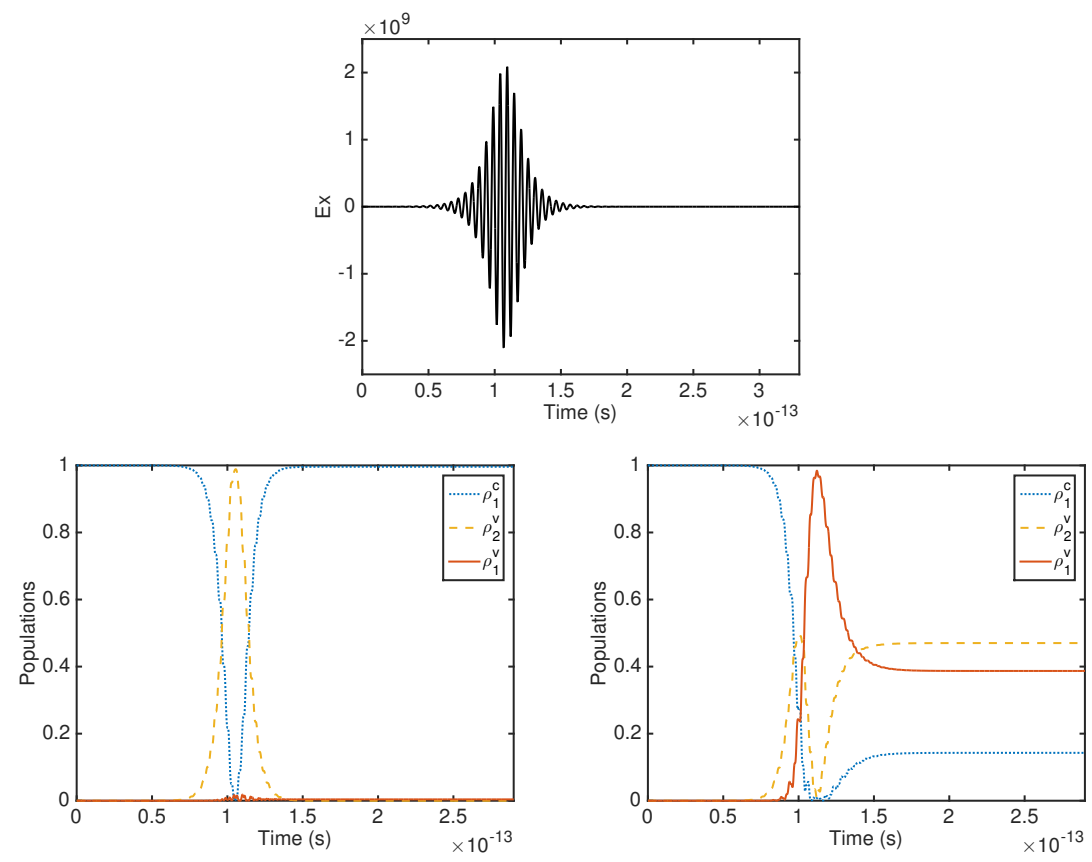

Fig. 2 Time evolution of $E_{x}$ (at the top) and populations (at the bottom) without e-ph interactions. Left: $\varepsilon_{2}^{v}-\varepsilon_{1}^{v}=2 \hbar \omega_{0}$; Right: $\varepsilon_{2}^{v}-\varepsilon_{1}^{v}=\hbar \omega_{0}$.

Now, we add the e-ph interactions. For simplicity, we assume that optical phonons are almost dispersionless and take a constant phonon energy $E_{\mathbf{q}}$ for the $N_{q}=100$ phonon modes that we consider. The time evolution of populations is presented in Fig. 3 for the test case in which $\varepsilon_{2}^{v}-\varepsilon_{1}^{v}=2 \hbar \omega_{0}$. We observe that e-ph interactions destroy the SIT phenomenon, even for valence levels far apart enough. In addition to a relaxation behavior, fast oscillations are generated for the two valence levels and persist after the electromagnetic pulse (as emphasized in the zoom presented in the right picture of Fig.3) . 

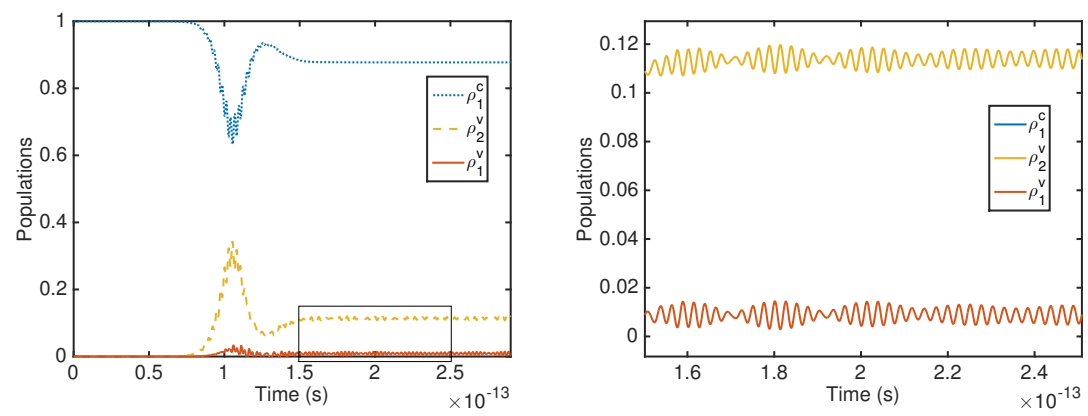

Fig. 3 Time evolution of populations for $\varepsilon_{2}^{v}-\varepsilon_{1}^{v}=2 \hbar \omega_{0}$ and $N_{\mathbf{q}}=100$ (left) and zoom inside the rectangle (right).

\section{Conclusion}

To study the interaction of quantum dots with an electromagnetic field taking into account e-ph interactions, we proposed an efficient discretization for the coupling appearing between the equation on the electron density $\rho$ and the set of equations on the phonon-assisted densities $S_{\mathbf{q}}$. For a better modeling, it will be now interesting to investigate how to take into account, via a kinetic equation, the quantum-well wetting layer into which quantum dots are embedded.

\section{References}

1. B. Bidégaray. Time discretizations for Maxwell-Bloch equations. Numerical Methods for Partial Differential Equations, 19(3):284-300, 2003.

2. B. Bidégaray-Fesquet. Positiveness and Pauli exception principle in raw Bloch equations for quantum boxes. Annals of Physics, 325(10):2090 - 2102, 2010.

3. B. Bidégaray-Fesquet and K. Keita. A nonlinear Bloch model for Coulomb interaction in quantum dots. Journal of Mathematical Physics, 55(2):021501, 2014.

4. H. Fröhlich. Electrons in lattice fields. Advances in Physics, 3(11):325-361, 1954.

5. E. Gehrig and O. Hess. Mesoscopic spatiotemporal theory for quantum-dot lasers. Phys. Rev. A, 65(033804):1-16, 2002.

6. H. Haug and S.W. Koch. Quantum theory of the optical and electronic properties of semiconductors. World Scientific, fifth edition, 2009.

7. T. Stauber, R. Zimmermann, and H. Castella. Electron-phonon interaction in quantum dots: A solvable model. Phys. Rev. B, 62(11):7336-7343, 2000.

8. G. C. Wick. The evaluation of the collision matrix. Phys. Rev., 80:268-272, 1950. 